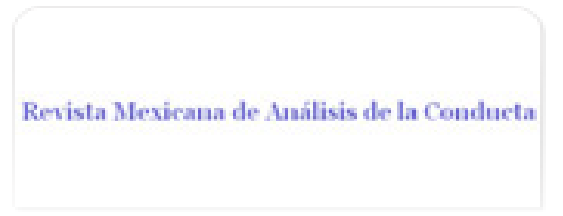

Revista Mexicana de Análisis de la Conducta ISSN: 0185-4534

editora@rmac-mx.org

Sociedad Mexicana de Análisis de la Conducta México

Espinosa Rodríguez, Julio; Santoyo Velasco, Carlos; Colmenares Vázquez, Ligia MEJORAMIENTO DE HABILIDADES DE ANÁLISIS ESTRATÉGICO DE TEXTOS EN ESTUDIANTES UNIVERSITARIOS

Revista Mexicana de Análisis de la Conducta, vol. 36, núm. 1, junio, 2010, pp. 63-84

Sociedad Mexicana de Análisis de la Conducta

Guadalajara, México

Disponible en: http://www.redalyc.org/articulo.oa?id=59313134005

- Cómo citar el artículo

- Número completo

- Más información del artículo

Página de la revista en redalyc.org

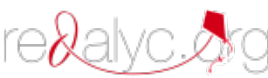

Sistema de Información Científica

Red de Revistas Científicas de América Latina, el Caribe, España y Portugal Proyecto académico sin fines de lucro, desarrollado bajo la iniciativa de acceso abierto 


\title{
MEJORAMIENTO DE HABILIDADES DE ANÁLISIS ESTRATÉGICO DE TEXTOS EN ESTUDIANTES UNIVERSITARIOS
}

\author{
IMPROVEMENT OF STRATEGICAL TEXT ANALYSIS ABILITIES IN \\ COLLEGE STUDENTS
}

\author{
JULIO ESPINOSA RODRÍGUEZ, CARLOS SANTOYO \\ VELASCO Y LIGIA COLMENARES VÁZQUEZ* \\ FACULTAD DE PSICOLOGÍA
}

UNIVERSIDAD NACIONAL AUTÓNOMA DE MÉXICO

\begin{abstract}
Resumen
Algunas de las habilidades y competencias esperadas en estudiantes de psicología por la Asociación Psicológica Americana (APA), pueden ejercitarse a través del abordaje estratégico de la literatura especializada en el campo. Estudiantes de primero, tercero y quinto semestres de la licenciatura en Psicología analizaron, mediante una guía basada en el Modelo de Análisis Estratégico de Textos, lecturas científicas de corte conductual. El programa siguió las siguientes etapas: (1) evaluación del repertorio de entrada, (2) modelamiento de la estrategia de análisis de textos y retroalimentación genérica del primer texto analizado, (3) utilización didáctica de las categorías del modelo en la docencia y (4) aplicación de dos nuevas situaciones de análisis de textos. En general, se muestra un avance generalizado en el dominio de las categorías del modelo en todos los grados escolares, así como niveles de ejecución cada vez más complejos. Esto se considera como evidencia de la validez ecológica del modelo y de la intervención. La validez social del instrumento se evaluó mediante un cuestionario que reflejó un alto nivel de satisfacción. Se discuten las implicaciones educativas del modelo para la formación de profesionales en Ciencias del Comportamiento.

Palabras clave: Análisis estratégico de textos, modelamiento, retroalimentación, habilidades metodológicas, estudiantes de Psicología




\begin{abstract}
Some of the abilities and skills expected by the American Psychological Association (APA) in the performance of undergraduate psychology students, may be developed and practiced through strategic comprehension of specialized literature. Undergraduate Psychology students of different levels analyzed three different scientific behavioral readings using the Strategic Text Analysis Model. The procedure included four stages: (1) diagnostic evaluation, (2) modeling and feedback of the text analysis strategy, (3) didactic use of the model in classes, and (4) two more evaluations of text analysis. General findings show a generalized progress in the adequate use of model categories for all groups, which is considered as evidence of the ecological validity of the Model. Students reported a high level of satisfaction suggesting also high social validity of the model. Educative implications of the strategy for the instruction of professionals in Behavioral Sciences are discussed.
\end{abstract}

Key words: Strategic analysis of texts, modeling, feedback, methodological abilities, Psychology students.

Este trabajo aborda la aplicación de un procedimiento de instrucción y evaluación basado en el Modelo de Análisis Estratégico de Textos (MAE txt) (Santoyo, 2001).

El procedimiento se sustenta básicamente en la Guía para la Certificación en Psicología de la American Psychological Association (APA), que refiere una diversidad de resultados de aprendizaje recomendados, sobre todo en lo concerniente a los cbnocimientos, habilidades y valores consistentes con la Ciencia y Aplicación de la Psicología (APA Guidelines for the Undergraduate Psychology Major, 2006). En esta experiencia formativa, se trabajó sobre aquellas habilidades y resultados de aprendizaje implicados en las tareas de análisis, evaluación y comprensión que forman parte del proceso activo de revisión de la literatura especializada y de los textos que los estưdiantes abordan en tres diferentes asignaturas del área básica de formación general. Partiendo del supuesto de que la mayoría de los textos científicos en ciencias del comportamiento $[\mathrm{CC}]$ cuentan con una estructura convencional que es factible de identificar y analizar, se asumió dicha estructura para el proceso de instrucción en la formación del profesional, convirtiendo el análisis y comprensión de textos en un conjunto de tareas explícitas con distintos niveles de complejidad el MAE txt, que facilitan a los estudiantes el aprendizaje, ejercicio y generalización de heurísticos o reglas genéricas (Gigerenzer \& Todd, 1999) para resolver situaciones que con frecuencia enfrentarán en el ámbito de su formación académica y del ejercicio profesional.

1 Se entiende por habilidad todas aquellas acciones o patrones de comportamiento que son producto del aprendizaje o educación y que nos capacitan a realizar adecuadamente otras actividades jerárquicamente y/o lógicamente asociadas.

que nos capacírna "convencional" para denotar los aspes disciplinios de unacomente asociadas. una convención asumida por la comunidad de expertos. 
Las habilidades implicadas en el MAE txt coinciden con los resultados centrales de aprendizaje propuestos en la Guía para la Certificación en tanto incorporan actividades con niveles de dificultad diversos pero que en su organización lógica se encuentran conectados armónicamente y constituyen un conjunto de convenciones aceptadas por la mayoría de los profesionales, enfoques disciplinarios e instituciones en las CC (ver el Manual de Publicaciones de la APA). En concreto, en un nivel básico, se espera que un profesional de las CC muestre habilidades como: la identificación de los objetivos de un estudio en su campo; la descripción de cómo los diseños de investigación abordan diferentes preguntas e hipótesis; la evaluación de las fuerzas y limitaciones de varios diseños y métodos. En un nivel más complejo se ubicarían habilidades como las de evaluar la validez y lo apropiado de las conclusiones del autor de la investigación e interpretar los resultados estadísticos básicos. En el contexto de este trabajo, se incorporaron además algunas otras habilidades: formular hipótesis verificables; elegir medidas válidas y confiables; generalizar apropiadamente las conclusiones de investigación basadas en los parámetros particulares de los métodos de investigación seleccionados; identificar componentes de los argumentos; distinguir entre supuestos, especulaciones 0 evidencias; identificar afirmaciones contradictorias o inapropiadas; evaluar la cualidad de las soluciones y revisarlas; seleccionar y conducir la mejor solución a un problema; demostrar habilidades orales y escritas de comunicación (APA Guidelines for the Undergraduate Psychology Major, 2006).

Considerando a la lectura como una tarea que requiere que los estudiantes movilicen distintas capacidades y competencias (Cepeda, 2008), se asume que los lectores expertos utilizan el conocimiento previo para darle sentido a la lectura, monitorean su comprensión durante el proceso lector, toman medidas para corregir errores de comprensión, leen con intención y asumen estrategias, resumen la información y hacen inferencias y preguntas durante y después de la lectura, lo que conlleva a distinguir lo relevante en los textos como una función de las metas que los lectores se han fijado (Pearson, Roehler, Dole \& Dufy, 2002). Por ello, en este trabajo se pretende instruir a los estudiantes en el manejo de una estrategia que les permita la comprensión de textos a través de un sistema de categorías isomórficas con las convenciones disciplinarias establecidas para la comunicación científica en psicología que además son componentes de competencias representativas básicas en la formación del psicólogo.

Una de las metas centrales de la formación profesional radica en que los resultados de aprendizaje sean generalizables a diferentes situaciones o dominios (Santoyo, 2001). Potencialmente, estas habilidades no sólo se ponen en práctica en las tareas de lectura sino que tienen implicaciones en el 
proceso de toma de decisiones ante problemáticas y situaciones diversas. La comparación bajo diferentes experiencias y contenidos realizada en el presente estudio permite generar una forma de evaluación para la ejecución de los estudiantes sobre un texto o un curso específico (Gottfried, Johnson \& Vosmik, 2009; Halonen, Bosack, Clay, McCarthy, Dunn, Hill, et al., 2003), y también analizar la generalidad de la estrategia y sus posibilidades como alternativa docente.

En términos generales, el MAE txt es una guía que solicita a los lectores ubicar y evaluar una serie de características esenciales de los textos científicos, para elaborar con ellas juicios y propuestas que aporten elementos de crítica y cursos alternativos de acción al tema científico en cuestión. EI MAE txt consta de nueve categorías isomórficas con la estructura genérica de textos disciplinarios en CC y otras áreas científicas; dichas categorías son relacionales en el sentido que se vinculan con los componentes más relevantes del texto que se está revisando. Al mismo tiempo, el modelo aprovecha la estructura lógica del texto para dirigir la atención del lector a los aspectos centrales y estratégicos del contenido para la comprensión del texto, derivando reglas o estrategias para la solución de problemas académicos, científicos y profesionales convencionales, a manera de heurísticos rápidos y adaptativos (Gigerenzer \& Todd, 1999).

Así, en la Tabla 1 se presentan las categorías del modelo, su definición y los niveles de ejecución mínimos requeridos para cada una de ellas. El nivel de ejecución se refiere a la tarea que deben realizar los alumnos con ese elemento del texto, que puede ser la identificación de algo textual, deducción a partir de otros elementos, análisis, evaluación, planeación o integración. Esa ejecución se evalúa en el producto escrito de los alumnos.

Una de las contribuciones más importantes del MAE txt al ámbito educativo se refiere a su uso como una herramienta instruccional y de evaluación sistemática del cambio conductual que se espera como producto de las experiencias formativas en la licenciatura en Psicología. A diferencia de otras estrategias didácticas en las que se evalúa el autorreporte del aprendizaje (Kerr \& Rynearson, 2004; Rizo, 2004; Taraban, Rynearson \& Kerr, 2000), o de aquéllas que en el afán de cuantificación, esquematizan y restringen las respuestas posibles que el estudiante puede emitir (Larson, Britt \& Larson, 2004; Rouet, Vidal-Abarca, Erboul, Millogo, 2001; Zarzosa, Luna, De Parrés \& Guarneros, 2007), el MAE txt permite categorizar y jerarquizar un amplio abanico de ejecuciones asociadas a problemas centrales de la metodología de la Psicología y a sus explicaciones como disciplina. 
Tabla 1.

Nivel de ejecución y definición condensada de las categorias del Modelo de Análisis Estratégico de Textos (Adaptada de Santoyo, 2001).

\begin{tabular}{|c|c|c|}
\hline Ejecución & Categoría de la Estrategia & Definición de la categoría \\
\hline $\begin{array}{l}\text { Identificación } \\
\text { o Deducción }\end{array}$ & Justificación Teórica (JT) & $\begin{array}{l}\text { Descripción de las razones } \\
\text { conceptuales para desarrollar el } \\
\text { trabajo. }\end{array}$ \\
\hline $\begin{array}{l}\text { Identificación } \\
\text { o Deducción }\end{array}$ & Justificación Metodológica (JM) & $\begin{array}{l}\text { Descripción de las razones técnicas } \\
\text { para desarrollar el trabajo }\end{array}$ \\
\hline $\begin{array}{l}\text { Identificación } \\
\text { o Deducción }\end{array}$ & Justificación Social (JS) & $\begin{array}{l}\text { Descripción del impacto o relevancia } \\
\text { social esperada del trabajo. }\end{array}$ \\
\hline $\begin{array}{l}\text { Identificación } \\
\text { o Deducción }\end{array}$ & Supuestos Básicos (SB) & $\begin{array}{l}\text { Premisas que regulan la aplicación } \\
\text { de la teoría y el método. La } \\
\text { explicación depende de ellas. }\end{array}$ \\
\hline Identificación & Objetivo $(\mathrm{O})$ & $\begin{array}{l}\text { Expresión de las metas del trabajo y } \\
\text { los medios para su consecución. }\end{array}$ \\
\hline $\begin{array}{l}\text { Identificación, } \\
\text { Deducción, } \\
\text { Análisis }\end{array}$ & Unidad de Análisis (UA) & $\begin{array}{l}\text { Propiedades del objeto de estudio en } \\
\text { virtud de las cuáles no puede } \\
\text { dividirse sin que su esencia se altere. }\end{array}$ \\
\hline $\begin{array}{l}\text { Identificación, } \\
\text { Deducción, } \\
\text { Evaluación }\end{array}$ & Estrategia Argumentativa (EA) & $\begin{array}{l}\text { Argumentos, críticas, antecedentes, } \\
\text { contradicciones presentados por el } \\
\text { autor para convencer al lector. }\end{array}$ \\
\hline $\begin{array}{l}\text { Identificación, } \\
\text { Deducción, } \\
\text { Evaluación }\end{array}$ & Estrategia Metodológica (EM) & $\begin{array}{l}\text { Conjunto de operaciones, diseño y } \\
\text { comparaciones para lograr el } \\
\text { objetivo. }\end{array}$ \\
\hline Evaluación & Coherencia Interna $(\mathrm{CI})$ & $\begin{array}{l}\text { Validez experimental interna, } \\
\text { coherencia de las secciones del texto. }\end{array}$ \\
\hline Evaluación & Coherencia Externa (CE) & $\begin{array}{l}\text { Validez experimental externa y } \\
\text { ecológica }\end{array}$ \\
\hline $\begin{array}{l}\text { Identificación, } \\
\text { Evaluación }\end{array}$ & $\begin{array}{l}\text { Evaluación de las Conclusiones del } \\
\text { Autor (CA) }\end{array}$ & $\begin{array}{l}\text { Relación lógica entre las } \\
\text { conclusiones del trabajo, objetivos, } \\
\text { estrategia y resultados }\end{array}$ \\
\hline Integración & Conclusión del Lector (CL) & $\begin{array}{l}\text { Impacto percibido del trabajo para el } \\
\text { lector }\end{array}$ \\
\hline $\begin{array}{l}\text { Evaluación y } \\
\text { Planeación }\end{array}$ & $\begin{array}{l}\text { Propuesta de Cursos Alternativos } \\
\text { de Acción (CAA) }\end{array}$ & $\begin{array}{l}\text { Propuestas del lector sobre nuevos } \\
\text { estudios y estrategias basadas en el } \\
\text { texto y la CL. }\end{array}$ \\
\hline
\end{tabular}

En ese sentido, MAE txt ha sido utilizado en diversos ámbitos de evaluación (p.e, Bazán \& García, 2002; Bazán, García \& Borbón, 2005; Espinosa \& Santoyo, 2007; Santoyo, Colmenares, Morales \& Flores 2005; Santoyo \& Sánchez, 1997; Villarreal, 2003;) aunque todavía son escasas las experiencias formativas publicadas al respecto.

En un ejemplo reciente, Cepeda (2008) evaluó distintas posibilidades de la estrategia de análisis de textos con cuatro grupos naturales de estudiantes del primer semestre de la carrera de Psicología encontrando que los estudiantes que recibieron instrucción en el MAE txt exhibieron avances importantes en su ejecución en el análisis de textos científicos con respecto a los estudiantes de grupos control sin instrucción explícita en tal modelo y de manera independiente a sus conocimientos metodológicos y habilidad verbal previos a la instrucción. 
Un objetivo importante en la evaluación de una estrategia de intervención se refiere a la validez social de la misma, es decir, el juicio de los participantes sobre el valor que tienen para ellos las metas de la intervención, el cambio en la conducta, el proceso utilizado para modificarla y los resultados (Wolf, 1978). Así, un propósito adicional en el presente estudio es la validación social de los resultados alcanzados por estudiantes que recibieron instrucción con base en el MAE txt en tres diferentes asignaturas ubicadas en distintos semestres, con lo que se pretende aportar no sólo indicadores de la generalidad del modelo a través de los distintos niveles de formación de los estudiantes, sino también evaluar la relevancia que perciben en relación con las distintas necesidades de la etapa formativa que cursan y si en función de la experiencia que han tenido con textos y tareas previas en Psicología, consideran que esta estrategia de lectura les es y/o será útil.

El propósito de este trabajo radica en evaluar los efectos del MAE txt como una alternativa docente equivalente para tres distintos cursos básicos de formación en $\mathrm{CC}$, sustentada en el modelamiento de la estrategia y la retroalimentación genérica de la ejecución de los estudiantes. Para cubrir con tal propósito se requiere de algunas condiciones básicas: un modelo de categorías asociadas con resultados centrales de aprendizaje propuestos en la Guía para la Certificación de la APA (el MAE txt), una estrategia didáctica que ofrezca oportunidades variadas para ejercitar las habilidades implicadas en el modelo, un contexto didáctico, una estrategia de retroalimentación de la ejecución de los estudiantes, y una forma de evaluación del impacto que la experiencia didáctica tiene en el establecimiento y consolidación de las habilidades de análisis de texto.

\section{Método}

\section{Participantes}

108 estudiantes de tres diferentes grupos naturales de semestres básicos del turno matutino de una licenciatura en Psicología, $78 \%$ de los cuales fueron mujeres y $22 \%$, hombres (ver Tabla 2 ).

Tabla 2 .

Datos generales de los participantes. Entre paréntesis se presenta el porcentaje de cada grupo en relación al total. El promedio de calificación del grupo de primer semestre corresponde al Bachillerato.

\begin{tabular}{lcccl}
\hline Semestre & N (\%) & $\begin{array}{c}\text { Promedio } \\
\text { Calificación }\end{array}$ & $\begin{array}{c}\text { Promedio } \\
\text { Edad }\end{array}$ & \multicolumn{1}{c}{ Asignatura } \\
\hline Primero & $37(34.25)$ & 8.53 & 18.6 & Introducción a la Psicología \\
Tercero & $44(40.74)$ & 8.39 & 19.6 & Aprendizaje y Memoria \\
Quinto & $27(25.00)$ & 8.58 & 20.1 & Psicología Experimental \\
Total & $\mathbf{1 0 8}(\mathbf{1 0 0 \% )}$ & $\mathbf{8 . 5 0}$ & $\mathbf{1 9 . 4 3}$ & \\
\hline
\end{tabular}




\section{Escenarios}

Las sesiones se condujeron en los salones de clase, que contaban con iluminación artificial y natural, pupitres individuales, un escritorio, un pizarrón y equipo para efectuar presentaciones audiovisuales.

\section{Materiales e instrumentos}

Presentación audiovisual (Power Point) del MAE txt (Santoyo, 1992; 2001), computadora portátil y equipo de videoproyección. La presentación muestra a los estudiantes la definición de las categorías del MAE txt, les brinda ejemplos generales de aplicación que son comunes en textos científicos en Psicología y modela la ejecución esperada con ejemplos concretos de aplicación basados en el texto que se utilizó como diagnóstico. La Figura 1 ejemplifica la presentación de la categoría Supuestos Básicos.

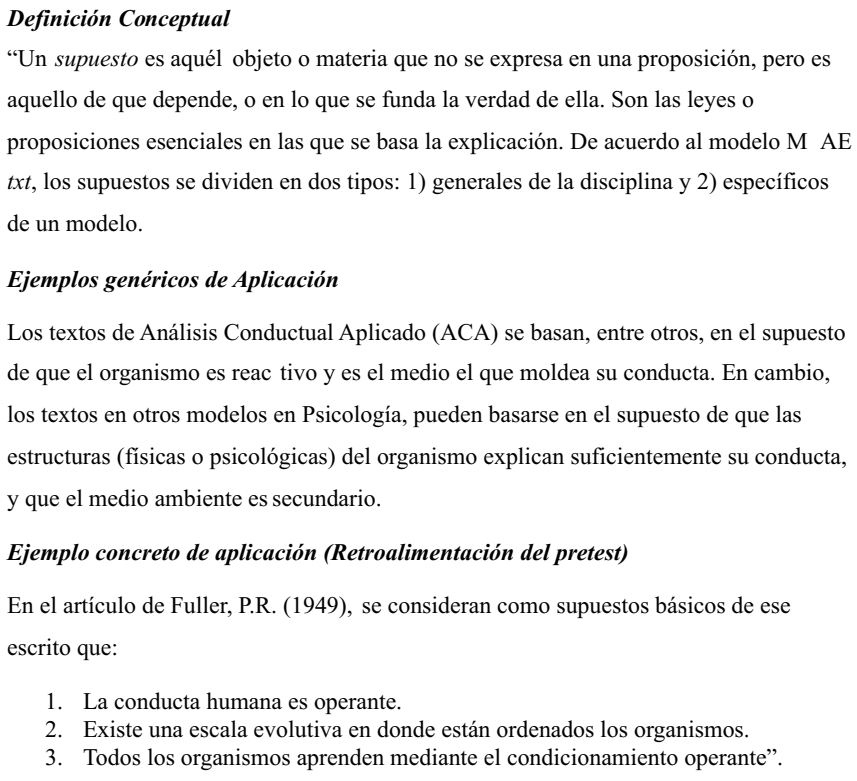

1. La conducta humana es operante.

2. Existe una escala evolutiva en donde están ordenados los organismos.

3. Todos los organismos aprenden mediante el condicionamiento operante".

Figura 1.Ejemplo de la presentación de las categorías del Modelo de Análisis de Textos. Se presenta el modelamiento de la categoría de Supuestos Básicos. 
Material de lectura. Se utilizaron tres reportes clásicos de investigación empírica en el campo del análisis de la conducta, publicados originalmente en prestigiadas revistas científicas. Los textos se seleccionaron por ser breves, susceptibles de ser analizados íntegramente en una sesión, y por encontrarse traducidos al español.

$\checkmark \quad$ Fuller, P.R. (1949). Condicionamiento operante de un organismo humano vegetativo. American Journal of Psychology, 62, 587-90.

$\checkmark \quad$ Baer, D.M. (1962). El control ejercido en el laboratorio sobre el chupeteo del pulgar mediante el retiro y la nueva presentación de reforzamiento. Journal of Experimental Analysis of Behavior, 5, 525-528.

$\checkmark \quad$ Ayllon, T. (1963). El tratamiento intensivo de la conducta psicótica por medio de la saciedad al estímulo y alimentos empleados como reforzadores. Behavior Research and Therapy, 1, 53-61.

Los artículos fueron tomados de la compilación de Ulrich, Stachnik, y Mabry (1974), y son equivalentes en cuanto a la estructura convencional de reporte de hallazgos científicos en Psicología, aunque como se muestra en la Tabla 3 difieren progresivamente en extensión y cantidad de información.

Tabla 3.

Estructura del material de lectura.

\begin{tabular}{|c|c|c|c|c|c|}
\hline Texto & Páginas & Palabras & Secciones & Experimentos & $\begin{array}{c}\text { Gráficos } \\
\text { o tablas }\end{array}$ \\
\hline Fuller, 1949 & 4 & 1657 & 1 & 1 & 0 \\
\hline Baer, 1962 & 6 & 2034 & 3 & 2 & 2 \\
\hline Ayllon, 1963 & 10 & 3718 & 16 & 3 & 3 \\
\hline
\end{tabular}

Formato de la Guía de Análisis de Textos con sección de Instrucciones y categorías a analizar en la forma de tareas o preguntas (verAnexo 1).

Instrumento de validación social. Adaptación del cuestionario de validación social diseñado ex profeso para evaluar el impacto de situaciones instruccionales en donde se utiliza el MAE txt (Santoyo, et al., 2005) (ver Anexo 2). 


\section{Diseño de investigación}

Para todos los grupos, el texto de Fuller sirvió para la evaluación diagnóstica. Posteriormente se realizó el modelamiento y retroalimentación, y dos aplicaciones posteriores de análisis de textos (artículos de Baer y Ayllon).

\section{Procedimiento}

En cada aplicación, los participantes leían un texto con la meta explícita de identificar, deducir o evaluar las categorías del MAE txt y con ello responder la guía de lectura. Todas las sesiones se hicieron dentro del horario asignado a cada asignatura, y el tiempo de cada sesión fue de aproximadamente 90 minutos, dependiendo de la extensión del texto por analizar. Esta actividad tenía un valor de $20 \%$ en las calificaciones parciales del curso.

Se inició con la evaluación diagnóstica a todos los grupos, antes de que los estudiantes recibieran instrucción acerca de las categorías y sobre la forma de trabajar con ellas.

Una vez que los estudiantes entregaron el producto del diagnóstico, cada docente realizó una presentación audiovisual del MAE txt, proporcionando retroalimentación y modelamiento durante aproximadamente una hora. La retroalimentación se realizó escuchando y discutiendo la pertinencia de las respuestas de los alumnos a las preguntas asociadas a cada categoría del análisis en el cuestionario. En una sesión posterior a la exposición audiovisual, se aplicó la prueba del segundo artículo y tres semanas después, se aplicó la tercera (ver Figura 2).

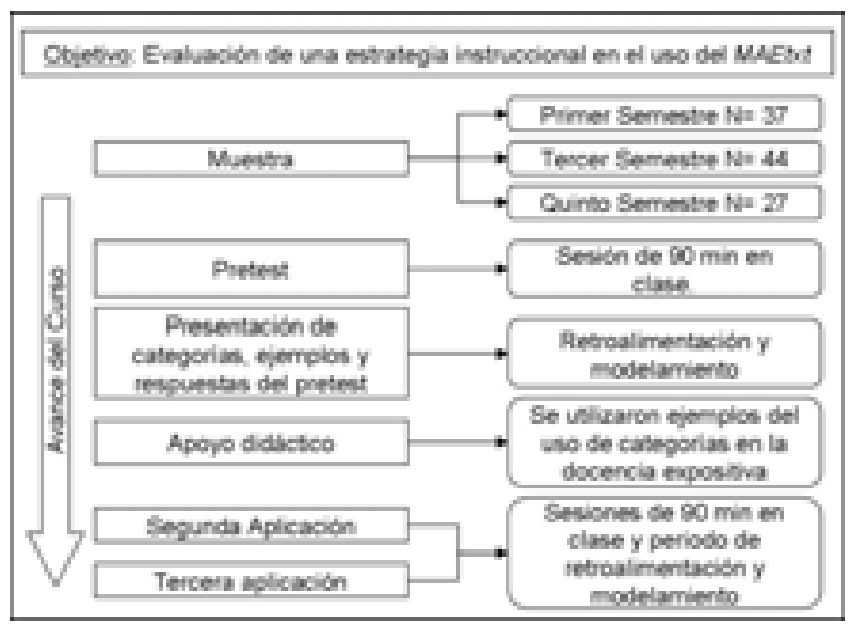

Figura 2. Diseño de la intervención. Estructura condensada del diseño y procedimiento. 
Se asignaron puntajes a las respuestas según los criterios que se presentan en la Tabla 4. Estos puntajes fueron el insumo principal para evaluar los avances en la ejecución de los alumnos. Además de la retroalimentación verbal presencial, los estudiantes recibieron sus puntajes individuales en cada ocasión como un indicador personalizado de su ejecución.

Tabla 4.

Criterios para evaluar el análisis de textos (Espinosa \& Santoyo, 2007).

\begin{tabular}{cl}
\hline Puntaje & \multicolumn{1}{c}{ Evaluación sobre la ejecución del participante } \\
\hline 0 & El participante no responde \\
1 & $\begin{array}{l}\text { Responde incorrectamente } \\
3\end{array}$ \\
& $\begin{array}{l}\text { Satisface el nivel de ejecución requerido en esa categoría } \\
\text { Responde de forma creativa, aportando elementos que van más } \\
\text { allá del texto literal (relaciona con otros aspectos del mismo } \\
\text { artículo, conecta con conocimiento previo, cuestiona sobre la base } \\
\text { de hipótesis creativas, etc.). }\end{array}$ \\
\hline
\end{tabular}

Si bien únicamente los artículos mencionados fueron considerados para los resultados de este estudio, los profesores de cada grupo utilizaron los contenidos de los programas de las asignaturas correspondientes para que en la exposición habitual se emplearan las categorías del MAE txt, modelando con ello la aplicabilidad del modelo frente a diferentes contenidos.

Después de la tercera evaluación, se aplicó el instrumento de evaluación de la validez social alcanzada, lo que permitió conocer la percepción de los participantes sobre la forma en que ellos realizaban el análisis de textos antes y después de la intervención (verAnexo 2). 


\section{Resultados}

Índice de Precisión Promedio. Se calculó él índice de precisión de los participantes, como el puntaje obtenido dividido entre 36, que es el puntaje que obtendrían al satisfacer el nivel de ejecución mínimo en las 12 categorías. En la Figura 3 se muestran los promedios de cada grupo en los tres artículos analizados. Conviene resaltar que más allá del promedio, después de la intervención aparecen algunos casos individuales que superan este criterio de "suficiencia" y obtienen puntajes de " 5 " (respuesta creativa) en una o varias categorías, siendo el caso de tres estudiantes en el análisis del segundo artículo y 16 para el tercero. Se observan incrementos en los puntajes de todos los grupos conforme aumenta el número de artículos analizados. También se aprecia que las diferencias entre los tres niveles de formación profesional se mantienen durante todo el estudio. El grupo de primer semestre pasó de tener un índice de precisión de 0.42 en el primer análisis, a uno de 0.81 en la tercera aplicación. El grupo de tercer semestre pasó de 0.53 a 0.85 , y el grupo de quinto semestre transitó de 0.55 a 0.95 . Estos datos son evidencia de una mejora sustancial generalizada en el desempeño progresivo de los participantes ante los análisis sucesivos que realizaron a lo largo del semestre. En el análisis relacionado con la variable de nivel o semestre escolar, se identificaron diferencias significativas para el primer artículo $(F=10.822 ; g l=2$, $105 ; p=<0.001$ ) entre el primer semestre y los otros dos, aunque no entre $3^{\circ} \mathrm{y}$ $5^{\circ}$; para el segundo artículo $(F=3.298 ; g l .2,105 ; p=0.041)$ se encontraron diferencias significativas entre los tres grupos; en el caso del tercer artículo también se observaron diferencias significativas $(F=6.173 ; g l .2,105 ; p=$ $0.003)$, aquí entre la ejecución de los grupos de primer y tercer semestre comparados con el grupo de quinto semestre.

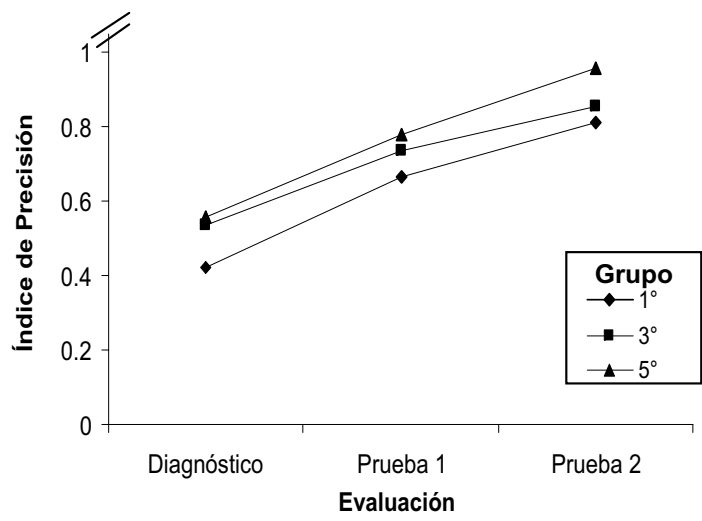

Figura 3. Índices de precisión. Promedio de cada grupo en los tres artículos analizados. 
El avance diferencial para cada una de las categorías que constituyen la Guía del MAE txt, se observa en la Figura 4. Las barras muestran el índice de precisión promedio para todos los participantes en cada una de las tres aplicaciones de la Guía (evaluación diagnóstica y dos seguimientos), y las líneas ilustran el rango de la distribución, es decir, la mínima y la máxima precisión entre los participantes.

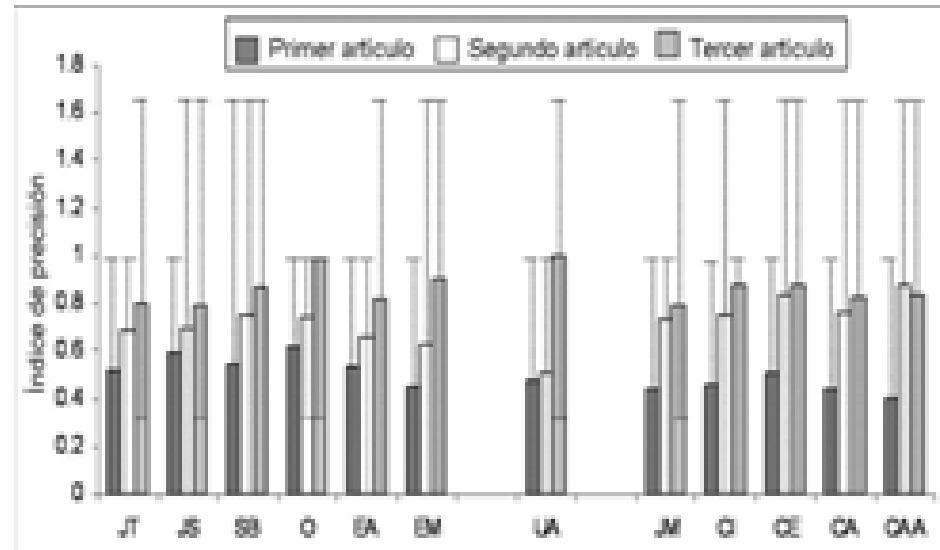

Figura 4.Índice de precisión promedio de todos los participantes para cada categoría de los tres artículos. Las líneas representan el rango.

Para efectos de comparación, se agruparon las categorías según las trayectorias de cambio que mostraron todos los estudiantes. Así, puede observarse que en las primeras seis tareas (JT, JS, SB, O, EA, EM), los estudiantes van avanzando progresivamente de una aplicación a la siguiente. En contraste, la categoría de Unidad de Análisis (UA), es estable entre el diagnóstico y el segundo análisis de artículos, aunque existe un avance significativo para la tercera aplicación. Por otra parte, las siguientes cinco categorías (JM, Cl, CE, CA, CAA), tienen un avance después de la retroalimentación y el modelamiento iniciales, para luego alcanzar un aparente nivel asintótico y sin cambios del segundo al tercer análisis. Es interesante el comportamiento de estas últimas categorías porque son precisamente las que implican niveles de ejecución más complejos: de evaluación, integración y planeación. También es notorio que se agrupe en este último bloque la categoría de Justificación Metodológica, que aparentemente compartiría niveles de dificultad con la Justificación Teórica y la Social. 
Los rangos mostrados permiten identificar diferencias individuales. Dentro de cada tarea de análisis de textos, los alumnos mostraron una diversidad de niveles de ejecución, que en muchos casos van desde un índice de precisión de cero (no responde en esa categoría) hasta índices superiores a 1, que implicarían respuestas que no sólo satisfacen el criterio mínimo de ejecución, sino que muestran un mayor dominio del tema, capacidad de relacionar conceptos, creatividad en la planeación, etc. Al igual que la media, los mínimos y los máximos van mejorando con la práctica en el análisis, y podemos constatarlo en que para el tercer texto ya no tenemos casos con puntajes de cero, y en que los índices superiores a 1 aparecen sobre todo en el segundo y tercer análisis.

En cuanto al desempeño académico de los participantes, el promedio que tenían los alumnos en el curso inmediato anterior no está asociado con la ejecución inicial en el análisis de textos ( $r$ de Pearson $=.11 ; p>.05)$. En cambio, la ejecución final en el análisis de textos predice significativamente el desempeño académico medido mediante la calificación final en la materia de referencia ( $r$ de Pearson $=.35 ; p<.001$ ), la cual era independiente de los ejercicios de análisis de texto realizados (pues cada profesor diseñaba sus propias evaluaciones conforme a los contenidos curriculares).

Validez Social. Mediante el cuestionario de validación social, se exploraron las descripciones de los participantes sobre su propio desempeño en cada una de las diversas categorias del MAE txt. Como se muestra en el Anexo 2, el instrumento les pedía elegir un porcentaje de dominio sobre cada una de las categorías, comparando el inicio del curso con el momento de la aplicación, que fue posterior al tercer análisis de textos. En la Tabla 5 se muestran los porcentajes promedio por categoría de análisis, para todos los grupos, antes y después del tratamiento. Los datos revelan un incremento generalizado en todas las categorías, sin embargo, una revisión más cuidadosa de los datos muestra ciertos matices en algunas categorías. 
En primer lugar, se observan porcentajes altos de inicio en categorías como objetivo y conclusiones de los autores. Esta condición tiene como consecuencia que los participantes también perciban un avance mayor en esas categorías, con porcentajes finales un poco arriba del $80 \%$. Este efecto se observa también en la categoría de justificación social. Por otra parte, los puntajes iniciales más bajos correspondieron al grupo del primer semestre.

Tabla 5 .

Porcentajes promedio de dominio percibido para cada una de las categorías del modelo, considerando dos momentos del curso.

\begin{tabular}{|c|c|c|c|c|c|c|}
\hline & \multicolumn{2}{|c|}{ 1er. Semestre } & \multicolumn{2}{|c|}{ 3er. Semestre } & \multicolumn{2}{|c|}{$5^{\circ}$ Semestre } \\
\hline & $\underline{\text { Inicio }}$ & $\underline{\text { Final }}$ & $\underline{\text { Inicio }}$ & $\underline{\text { Final }}$ & $\underline{\text { Inicio }}$ & $\underline{\text { Final }}$ \\
\hline Justificación Teórica & 23.6 & 66.2 & 35.0 & 63.6 & 41.0 & 74.0 \\
\hline Justificación Metodológica & 25.7 & 67.6 & 27.8 & 61.9 & 28.7 & 66.7 \\
\hline Justificación Social & 30.4 & 72.3 & 36.4 & 67.6 & 39.8 & 82.4 \\
\hline Supuestos Básicos & 20.9 & 64.2 & 23.3 & 62.5 & 33.3 & 65.7 \\
\hline Objetivo & 43.9 & 79.0 & 48.9 & 76.1 & 52.8 & 80.6 \\
\hline Unidad de Análisis & 29.6 & 74.3 & 26.1 & 66.5 & 45.4 & 79.6 \\
\hline Estrategia Argumentativa & 25.0 & 70.9 & 21.0 & 51.7 & 25.0 & 61.5 \\
\hline Estrategia Metodológica & 25.7 & 69.6 & 26.1 & 56.8 & 32.4 & 66.7 \\
\hline Coherencia Interna & 25.1 & 69.6 & 26.7 & 62.5 & 28.7 & 62.0 \\
\hline Coherencia Externa & 21.0 & 73.0 & 28.4 & 60.8 & 25.0 & 58.3 \\
\hline Conclusiones del Autor & 49.3 & 81.8 & 45.4 & 76.1 & 53.7 & 81.5 \\
\hline Conclusiones Alternativas & 23.6 & 68.9 & 27.3 & 63.6 & 32.4 & 70.6 \\
\hline Promedio & 28.7 & 71.4 & 31.0 & 64.2 & 36.5 & 70.8 \\
\hline
\end{tabular}

A pesar de que se trata de un nivel de análisis diferente a la ejecución, esta medida de validez social converge significativamente con los resultados de los análisis de textos, pues la percepción sobre el dominio inicial en todas las categorías correlaciona con el puntaje obtenido en el diagnóstico ( $r$ de Pearson $=0.22 ; p=.01$ ), y sin embargo, no muestra relación con el promedio obtenido en el curso inmediato anterior. Asimismo, la percepción sobre el nivel de dominio al final del proceso correlaciona con el puntaje obtenido en el análisis del último artículo ( $r$ de Pearson= $0.26 ; p=.004)$. 


\section{Discusión}

En este trabajo se ha desarrollado una experiencia docente apoyada en el MAE txt, cuyos fundamentos teóricos y didácticos han sido expuestos con anterioridad (Santoyo, 1992, 2001) y que en esta ocasión, ha puesto a prueba su validez ecológica en la práctica formativa de los psicólogos. Dada la escasez de estudios empíricos al respecto, los resultados obtenidos son prometedores. En un contexto en donde existe una demanda para el desarrollo de competencias, más que en el mero conocimiento de contenidos (Castañeda, 2004), se hace necesario enfatizar un aspecto fundamental: el análisis estratégico de los contenidos incluidos en los textos. Esta visión implica que los estudiantes sean activos en el uso de estrategias para analizar y comprender mejor un texto, pero también supone que tales estrategias pueden ser modificadas por experiencias diseñadas ex profeso.

Los resultados demuestran la maleabilidad de las habilidades implicadas por la estrategia de análisis y un impacto positivo sobre la satisfacción de los usuarios.

Los estudiantes de los tres diferentes niveles mostraron avances significativos en el dominio del MAE txt en diferentes lecturas. Un aspecto importante del desempeño exhibido radica en que los participantes de los tres grupos lograron avances progresivos con cada aplicación, siendo notable que los progresos alcanzados por los estudiantes de semestres iniciales igualan en la segunda aplicación los puntajes obtenidos por los estudiantes de los semestres avanzados en la primera aplicación. Ese mismo efecto se obtuvo globalmente en la tercera aplicación para toda la muestra. Las diferencias en ejecución atribuibles al nivel de estudios (semestres cursados) se mantienen relativamente constantes para los semestres primero y quinto.

Dadas las categorías de codificación de la ejecución de los estudiantes para todos los niveles de comprensión seleccionados, la ejecución exhibida al final de la experiencia implica que los participantes lograron un dominio en la identificación adecuada de los componentes requeridos del texto con base al MAE txt, logro sustantivo que muestra que las habilidades asociadas a las categorías del MAE txt son maleables a través de experiencias basadas en modelamiento, práctica y retroalimentación. Los resultados de este estudio confirmaron que las habilidades adquiridas se aplican de manera equivalente y progresivamente más compleja, a pesar de las diferencias formales de los textos. 
El grado de avance encontrado en este estudio es relativamente similar al obtenido por Cepeda (2008), con materias afines en otro campus universitario.

Por otra parte, si bien se muestra la maleabilidad de las habilidades implicadas en el MAE txt el dominio mostrado sólo alcanzó el nivel de identificación y uso adecuado de las categorías. La frecuencia de respuestas creativas exhibidas fue relativamente baja y éstas fueron exhibidas sólo por unos cuantos participantes durante la segunda y la tercera aplicación. Este hecho implica dos posibilidades: que con práctica adicional se podría lograr una ejecución con mayor cantidad de respuestas creativas, o que se requieren estrategias de apoyo dirigidas explícitamente a inducir respuestas creativas de parte del estudiante, mediante cuestionamientos, instigación o demandas expresas al respecto, como previamente intentaron Santoyo, et al (2005) quienes, una vez detectado el nivel de ejecución exhibido en el análisis, con base en retroalimentación positiva, inducían a los alumnos con diversas sugerencias abiertas para acceder al nivel de ejecución inmediato superior en una escala de evaluación diseñada ex profeso. No obstante, se requiere de más estudios que prueben y extiendan dichas posibilidades.

La experiencia instruccional que se reporta se apoya en un modelo poco evaluado pero de potencial utilidad como herramienta de apoyo a las prácticas docentes como instrumento que permita identificar y solventar déficits de comprensión lectora.

De acuerdo con Gigerenzer y Todd (1999) ante la gran complejidad de información a la que nos enfrentamos, aprendemos a utilizar heurísticos, reglas rápidas y expeditas que garanticen altas probabilidades de éxito en la solución de los problemas más recurrentes en nuestro ambiente. EI MAE txt busca ser congruente con lo anterior y con las ideas del contextualismo o perspectivismo de McGuire (1999) ofreciendo vías de investigación educativa para futuras innovaciones que permitan a los alumnos utilizar aquellas reglas que respondan a las convenciones disciplinarias implícitas y explícitas en los artículos de investigación que enfrentan cotidianamente.

Algunos autores (Castañeda, 2004) proponen que una de las metas principales de la formación académica sería mejorar el razonamiento de los estudiantes. En este trabajo asumimos que el desarrollo de estas habilidades (Santoyo \& Cedeño, 1986) asociadas al análisis estratégico de textos es una alternativa para cubrir dichas metas. 
Si bien la experiencia global a la que se expusieron los estudiantes fue productiva y se alcanzaron avances importantes en su ejecución, se requiere de mayor investigación que dé cuenta de las diferencias individuales en tanto la ejecución inicial, el grado diferencial de avance y la convergencia o divergencia entre la percepción inicial respecto a la percepción final y su relación con la ejecución final, aspectos a explorar con seguimientos semestrales en futuros estudios.

Los datos sobre la validación social de la estrategia reflejan una aceptación generalizada sobre los procedimientos seguidos, pues los usuarios describen avances de cerca del doble respecto al nivel en que percibían en un inicio sus propias habilidades asociadas a cada categoría del modelo, aunque se observan cambios diferenciales por categoría y por grado. Por ejemplo, en algunas categorías como objetivo y conclusiones de los autores, los puntajes iniciales ya eran altos, probablemente porque en la mayoría de los artículos de corte empírico esta información es explícita. También es notorio que la percepción de dominio y beneficio es aún mayor para los estudiantes del primer semestre. Sin embargo, todos los estudiantes reconocen que todavía queda un buen trayecto por avanzar, lo cual aumenta las probabilidades de adherencia al modelo a partir de que ellos mismos observan consecuencias positivas de su uso. Es relevante la relativa convergencia entre las medidas de ejecución y las de percepción, pues sugiere que los estudiantes son capaces de evaluar su desempeño y son relativamente sensibles a los cambios en sus competencias.

Por otra parte, el que los usuarios perciban la experiencia con el modelo como favorable y productiva para su formación académica y profesional sugiere que es más probable que se adhieran al empleo del modelo para el estudio cotidiano o al menos para la elaboración de trabajos solicitados frecuentemente en las asignaturas teóricas y en particular en los aspectos prácticos (p.e., revisiones monográficas, elaboración de proyectos de evaluación, intervención o investigación). La percepción de auto-eficacia en el empleo del MAE txt, convierte a la experiencia en una variable disposicional o motivacional (Corral, 1997) que conviene fomentar en la experiencia académica y formativa de los estudiantes en Ciencias del Comportamiento.

En conclusión, en este trabajo se asume al lector, conceptualmente, como un ente activo que interactúa con el texto con base en sus conocimientos previos y que es capaz de utilizar guías categóricas para producir una mejor comprensión. 
Así, el lector identifica y analiza conceptos y estrategias para una eventual evaluación y derivar de ello actividades o metas en otro nivel (p.e., fundamentar una pregunta de investigación original y la estrategia asociada a su solución). Esta ejecución puede darse a distintos niveles de complejidad, y es posible exponer a quienes se acercan a las CC, a las experiencias y la retroalimentación necesarias para que puedan transitar a niveles cada vez más expertos. Dicha interacción entre el lector y los textos se postula como un resultado deseable en la perspectiva de dos contextos posibles. Uno de ellos es el disciplinario, que asume a las explicaciones científicas como relativas y perfectibles (Mc Guire, 1999) y en donde el estudiante en formación desarrolla habilidades que le permiten ir más allá de recibir información, y ser capaz de delimitar y extender los alcances de su disciplina. El otro contexto es el de las demandas del ambiente académico o laboral; ambos imponiendo restricciones, pero también induciendo constantemente el planteamiento de cursos de acción alternativa en la tarea de comprensión de textos para edificar justificaciones y argumentos, para la construcción de explicaciones y para la solución de problemas de relevancia social, profesional, metodológica o teórica (Santoyo, 2001).

\section{Referencias}

American Psychological Association (2006). APA guidelines for the undergraduate psychology major. Washington, DC: Recuperado el 2 de junio de 2009 de: www.apa.org/ed/resources.html

Baker, L., \& Brown, A.L. (1984). Metacognitive skills and reading. En P.D. Pearson, R. Barr, M.L. Kamil \& P. Mosenthal (Eds.), Handbook of reading research (pp. 353-394). New York. Longman.

Bazán, A., \& García, I. (2002). Relación estructural de indicadores de ingreso y permanencia y el dominio de habilidades metodológico-conceptuales en cuatro grupos de estudiantes de educación. Revista Latinoamericana de Estudios Educativos, 32, 2, 105-122.

Bazán, A., García, I., \& Borbón, J.C. (2005). Evaluación de habilidades metodológico conductuales en el análisis de textos científicos. Algunos hallazgos científicos. En Santoyo, C. (Ed.), Alternativas Docentes III. Análisis y evaluación de las habilidades metodológicas, conceptuales y profesionales en la formación del Psicólogo. México: Facultad de Psicología: UNAM.

Castañeda, F. S. (2004). Competencias del recién egresado de la licenciatura en Psicología. Psicología desde el Caribe, 13, 109-143.

Cepeda, I.M.L. (2008). Estrategia de comprensión de textos científicos en estudiantes de Psicología: Un estudio de evaluación e intervención. Tesis de Doctorado en Educación. Universidad Anáhuac. 
Corral, V.V. (1997). Un análisis crítico del concepto "actitudes": Parte I: Postulados y métodos de estudio. Revista mexicana de análisis de la conducta, 23, 3, 215-236.

Espinosa, R.J., \& Santoyo, V.C. (2007). Evaluación de una estrategia para el análisis de textos científicos: un estudio transversal. Ponencia presentada en el XVIII Congreso Mexicano de Análisis de la Conducta, Xalapa, Ver.

Flavell, J. H. (1979). Metacognition and cognitive monitoring: A new area of cognitive-developmental inquiry. American Psychologist, 34, 906-911.

Gigerenzer, G., \& Todd, P.M. (1999). Simple heuristics that make us smart. New York: Oxford University Press.

Gottfried, G.M., Johnson, K.E., \& Vosmik, J.R. (2009). Assessing student learning: a collection of evaluation tools. Office of Teaching Resources in Psychology. Recuperado el 22 de julio de 2009 de OTRP Online, sección Teaching Resources/ Research and Teaching: http://teachpsych.org/otrp/resources/gottfried09.pdf

Halonen, J., Bosack, T., Clay, S., McCarthy, M., Dunn, D. S., Hill, IV G. W., McEntarffer, R., Mehrotra, C., Nesmith, R., Weaver, K. A., \& Whitlock, K. . (2003). A rubric for learning, teaching, and assessing scientific inquiry in psychology. Teaching of Psychology, 30, 196-208.

Kerr, M., \& Rynearson, K. (2004). Analytic and pragmatic factors in college students' metacognitive reading strategies, Reading Psychology, 25, 6781.

Larson, M., Britt, M. A., \& Larson, A. A. (2004) Disfluencies in comprehending argumentative texts, Reading Psychology, 25, 205-224

McGuire, W.J. (1999). Creative hypothesis generating in psychology. Annual Review of Psychology, 48, 1-30.

Pearson, P.D., Roehler, L.R., Dole, J.A., \& Duffy, G.A. (2002). Developing expertise in reading comprehension. En S.J. Samuels \& A.E., Fastrup (Eds.). What research has to say about reading instruction. (pp. 145-199). Newark, D.E.: IRA.

Rouet, J.F., Vidal-Abarca, E., Erboul, A. B., \& Millogo, V. (2001). Effects of information search on the comprehension of instructional text, Discourse Processes, 31 (2), 163-186.

Rizo, P.M. (2004). Programa de instrucción para desarrollar estrategias para la comprensión y el aprendizaje de textos escritos, Revista Latinoamericana de Estudios Educativos, 34 (2), 113-137. 
Santoyo, V.C. (2001). Estrategias de avance en comprensión, análisis y evaluación de textos científicos y profesionales. En C. Santoyo (comp.). Alternativas Docentes, Volumen II: Aportaciones al estudio de la formación en habilidades metodológicas y profesionales en las ciencias del comportamiento (pp. 9-40). México, D.F.: PAPIME/UNAM.

Santoyo, V.C. (1992). El análisis de las habilidades científicas y profesionales: Las aportaciones del enfoque contextual. Revista Sonorense de Psicología, 6, 665-73.

Santoyo, V.C., Colmenares, V.L., Morales, Ch. S. \& Flores, M. N. (2005). Una experiencia instruccional basada en el modelo de análisis estratégico de textos en psicología conductual. Ponencia presentada en el XVII Congreso Mexicano de Análisis de la Conducta. San Luis Potosí, SLP.

Santoyo, V.C., Morales, Ch., S., Colmenares, V.L., \& Flores, M.N. (2005). Validación social del modelo de análisis estratégico de textos. Ponencia presentada en el XVII Congreso Mexicano de Análisis de la Conducta. San Luis Potosí, SLP.

Santoyo, V.C., \& Sánchez, J.M. (1997). Una estrategia para evaluar habilidades científicas y profesionales: La experiencia de la Facultad de Psicología. En Comisión para el Cambio Curricular (Editores). Hacia el cambio curricular: Diagnóstico del currículum actual de la Facultad de Psicología (pp. 1057-1093). México: Facultad de Psicología, UNAM.

Taraban, R. M., Rynearson, K., \& Kerr, M. (2000). College students' academic performance and self- reports of comprehension strategy use, Reading Psychology, 21, 283-308.

Ulrich, R., Stachnik, T., \& Mabry, J. (1974). Control de la conducta humana: Modificación de la conducta aplicado al campo de la educación. México: Trillas. Vol. III.

Villarreal, V. G. (2003). Un ejercicio diagnóstico: la detección de un perfil de ingreso a un doctorado en Psicología. Tesis de Licenciatura en Psicología. Facultad de Psicología, UNAM.

Wolf, M.M. (1978). Social validity: The case for subjective measurement or how applied behavior analysis is finding its heart. Journal of Applied Behavior Analysis, 11, 203-214.

Zarzosa, E. L., Luna, P. D., De Parrés, F. T., y Guarneros, E. (2007). Efectividad del uso de una interfaz para la lectura estratégica en estudiantes universitarios. Un estudio exploratorio. Revista Electrónica de Investigación Educativa, 9 (2). Recuperado el 08 de marzo de 2009: http://redie.uabc.mx/vol9no2/contenidozarzosa.html 


\section{ANEXO 1: GUÍADEANÁLISIS DETEXTOS}

\section{INSTRUCCIONES}

"El presente ejercicio constituye una situación de evaluación diagnóstica respecto de la forma cómo analizan textos científicos los estudiantes de la Facultad de Psicología. A continuación se te presentará un texto de investigación psicológica en el que deberás responder a una serie de preguntas una vez que hayas leído y comprendido el texto. Podrás regresar al texto cuantas veces consideres necesario para responder a las preguntas.

Con la finalidad de optimizar recursos, se te solicitará no escribas en el texto del artículo, para ellos se te proporcionará una hoja de respuestas para que escribas en el espacio correspondiente la respuesta que consideres concuerda con la información solicitada".

"Debido a que se considera que la habilidad de análisis estratégico de textos científicos en Psicología es una herramienta fundamental en la formación académica del Psicólogo, los docentes participantes podrían estimular, con una puntuación adicional a la calificación parcial dentro del curso, los productos concluidos de los estudiantes".

"Escribe dentro del espacio indicado la respuesta solicitada. El ejercicio requiere respuestas concretas, por lo que se te solicita integres tus respuestas en forma breve y sustantiva en el lugar correspondiente. Si tienes alguna duda respecto a lo que se te está solicitando, puedes preguntar al docente responsable".

\section{HOJADE RESPUESTAS}

1. Describe cuál es la relevancia del trabajo (aportación principal propuesta por el autor) en cuanto a la:

Justificación teórica

Justificación metodológica

Justificación Socia

2. Describe cuáles son los supuestos principales de los que derivan las explicaciones y argumentaciones en las que se sustenta el trabajo.

3. Describe cuál es el objetivo general del artículo.

4. Describe cuál es la Unidad de Análisis (objeto de estudio o variable dependiente).

5. Los autores generalmente usan dos clases de estrategias, una para argumentar y convencernos de lo valioso de su trabajo y otra para desarrollar los procedimientos para obtener la información que permita llegar a conclusiones. A continuación describe:

La estrategia argumentativa

La estrategia metodológica

6. ¿El trabajo es coherente y válido en cuanto a lo propuesto y los resultados?, describe porqué.

7. ¿El trabajo es coherente y válido en cuanto a sus resultados y lo que dice la literatura? ¿se podrá generalizar?, describe porqué

8. Describe si la conclusión o conclusiones del autor son consistentes y valiosas.

9. Todo trabajo es perfectible; ¿qué recomendaciones harías para mejorar o hacer más valioso el trabajo que has leído? 


\section{ANEXO 2: CUESTIONARIO DE VALIDACIÓN SOCIAL}

\section{CUESTIONARIO DE SATISFACCIÓN: ANÁLISIS ESTRATÉGICO DE TEXTOS}

Sexo (H) (M) Edad

Área (s) de interés
Semestre

Promedio

Instrucciones. A continuación se presentan una serie de preguntas que te pedimos que respondas con la mayor honestidad posible para saber que tan útil y efectiva ha sido la estrategia para analizar textos que aprendiste a lo largo del semestre. A partir de este cuestionario tendremos la posibilidad de mejorar el modelo. Si tienes dudas pregunta al Profesor. Si estás listo (a) comienza a responder el cuestionario ahora.

En la siguiente tabla, marca con una $\mathbf{X}$ el porcentaje de ocasiones en las que antes del curso eras capaz de realizar las siguientes actividades con los textos que revisabas y cuál es tu capacidad actualmente.

\begin{tabular}{|c|c|c|c|c|c|c|c|c|c|c|}
\hline \multirow{3}{*}{ HABILIDADES } & \multicolumn{5}{|c|}{ Al inicio del curso } & \multicolumn{5}{|c|}{ En este momento } \\
\hline & \multicolumn{10}{|c|}{ Nivel } \\
\hline & $0 \%$ & $25 \%$ & $50 \%$ & $75 \%$ & $100 \%$ & $0 \%$ & $25 \%$ & $50 \%$ & $75 \%$ & $100 \%$ \\
\hline \multicolumn{11}{|l|}{$\begin{array}{l}\text { Justificación } \\
\text { Teórica }\end{array}$} \\
\hline \multicolumn{11}{|l|}{$\begin{array}{l}\text { Justificación } \\
\text { Metodológica }\end{array}$} \\
\hline \multicolumn{11}{|l|}{ Justificación Social } \\
\hline \multicolumn{11}{|l|}{ Objetivos } \\
\hline \multicolumn{11}{|l|}{ Supuestos Básicos } \\
\hline \multicolumn{11}{|l|}{ Unidad de análisis } \\
\hline \multicolumn{11}{|l|}{$\begin{array}{l}\text { Estrategia de } \\
\text { Argumentación }\end{array}$} \\
\hline \multicolumn{11}{|l|}{$\begin{array}{l}\text { Estrategia } \\
\text { Metodológica }\end{array}$} \\
\hline \multicolumn{11}{|l|}{$\begin{array}{l}\text { Coherencia } \\
\text { Interna }\end{array}$} \\
\hline \multicolumn{11}{|l|}{$\begin{array}{l}\text { Coherencia } \\
\text { Externa }\end{array}$} \\
\hline \multicolumn{11}{|l|}{ Conclusión } \\
\hline $\begin{array}{l}\text { Cursos alterna tivos } \\
\text { de acción }\end{array}$ & & & & & & & & & & \\
\hline
\end{tabular}

